\title{
Non-visual analysis of miniaturized melt wire arrays for in-pile measurement of peak irradiation temperature
}

Kunal Mondal, Kiyo Fujimoto and Michael McMurtrey

February, 2020

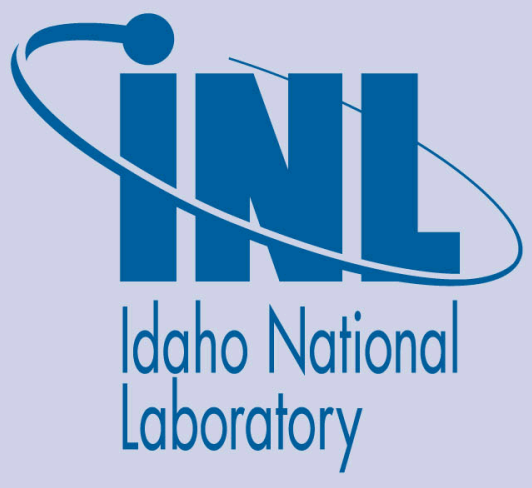




\section{DISCLAIMER}

This information was prepared as an account of work sponsored by an agency of the U.S. Government. Neither the U.S. Government nor any agency thereof, nor any of their employees, makes any warranty, expressed or implied, or assumes any legal liability or responsibility for the accuracy, completeness, or usefulness, of any information, apparatus, product, or process disclosed, or represents that its use would not infringe privately owned rights. References herein to any specific commercial product, process, or service by trade name, trade mark, manufacturer, or otherwise, does not necessarily constitute or imply its endorsement, recommendation, or favoring by the U.S. Government or any agency thereof. The views and opinions of authors expressed herein do not necessarily state or reflect those of the U.S. Government or any agency theof. 


\section{Non-visual analysis of miniaturized melt wire arrays for in-pile measurement of peak irradiation temperature}

Kunal Mondal, Kiyo Fujimoto and Michael McMurtrey

February, 2020

Idaho National Laboratory Idaho Falls, Idaho 83415

http://www.inl.gov

Prepared for the

U.S. Department of Energy Office of

Under DOE Idaho Operations Office

Contract DE-AC07-05ID14517 


\begin{abstract}
As a part of the in-pile instrumentation program, the Idaho National Laboratory (INL) has established in-house capabilities to fabricate and test new advanced manufactured printed sensors for measurement of peak temperature inside the harsh environment of a nuclear test reactor. Although nuclear test reactors may use standard methods that can provide real-time temperature information, such as thermocouples, the need for feedthroughs for real-time measurements greatly increases the cost of the experiment. Some experiments also present the problem of limited space available for instrumentation. To address this need, INL is working to improve temperature detection options for such applications. The lab is developing advanced manufactured melt wire arrays to determine peak temperatures in experiments with limited space. Furthermore, capabilities to print multiple melt wires with different melting properties on a single miniaturized unit is being established. Recent results and knowledge from this ongoing work have made it possible to develop unique temperature sensors that can accomplish user specific temperature ranges inside the harsh environments experienced during radiation testing
\end{abstract}




\section{CONTENTS}

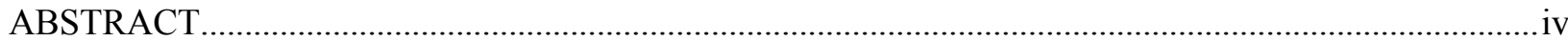

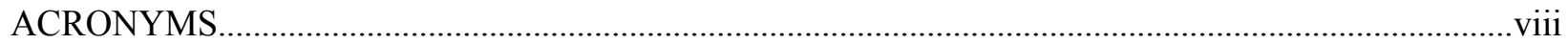

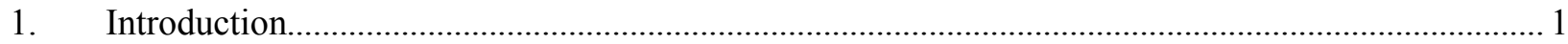

2. Encapsulation of printed melt wires....................................................................................... 2

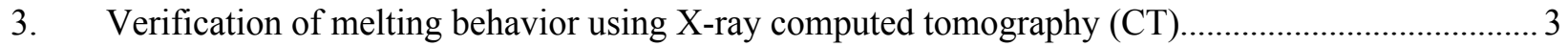

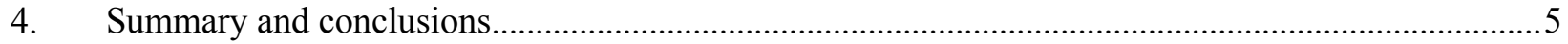

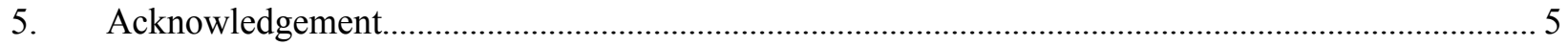

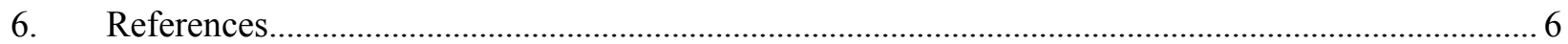

\section{FIGURES}

Figure 1. Schematic illustrating the melt wire printing and encapsulation. 1

Figure 2. Printing of melt wires: (a) blank steel chip, (b) printed silver melt wires. (c) profilometric analysis of melt wires before sandwiching with a plane steel lid to estimate width and height of the wire, (d) actual $2 \mathrm{~mm}$-diameter melt wire sensor chip. The inset shows clear view of laser welded chip.

Figure 3. Characterization of printed melt wires. X-ray computer tomography (CT scan) of the melt wires sensor confirms the morphology of the printed line. Inset shows the actual printed chip

Figure 4. Characterization of printed melt wires (a) X-ray computer tomography (CT scan) of the melt wires sensor to confirm that the line is unharmed due to laser welding. Furnace test of the melt wire: (b) before heating, and (c) after heating at $1000{ }^{\circ} \mathrm{C}$ confirms melting of the printed wire.

Figure 5. Optical characterization of printed melt wires: (a) before melting. (i) the printed chip, (ii) the clear view and (iii) dimensions of the printed wire using profilometry. (b) after melting due to heating at $1000{ }^{\circ} \mathrm{C}$ confirms the melting. (i) printed chip after melting, (ii) the clear view and (iii) dimensions of the melted portion of the wire using profilometry..... 


\section{ACRONYMS}

$\mathrm{AM}$ - Advanced manufacturing

ATR - Advanced Test Reactor

AJP - Aerosol Jet Printing

CT - Computed tomography

GE - General Electric

nXCT - Nano-focused X-ray computed tomography

PJP - Plasma Jet Printing

$\mathrm{SS}$ - Stainless steel 


\section{Non-visual analysis of miniaturized melt wire arrays for in-pile measurement of peak irradiation temperature}

\section{Introduction}

The harsh environment inside nuclear reactors creates a challenging problem for a variety of materials from fuels to structural materials. Researchers are working to better understand how this environment affects materials and components, particularly the effect of neutron irradiation, which is unique to the nuclear field. Test reactors, such as the Advanced Test Reactor at Idaho National Laboratory, provide researchers a means to study the effects of neutrons in their experiments. While some experiments need only to study the material with post-irradiation examination to complete research objectives, others require information during the irradiation inside the reactor. As a result, significant research and development is ongoing to allow for increased instrumentation in nuclear test reactors.

The Advanced Manufacturing (AM) portion of the In-Pile Instrumentation program has been exploring novel methods for producing unique sensors that are not manufacturable using traditional means. Direct ink writing techniques such as aerosol jet printing (AJP) and plasma jet printing (PJP), were identified as potential tools with applications across various sensor types. They are capable of creating complex 2D designs using a variety of materials and have demonstrated significant potential for the development of innovative in-pile instrumentation. These techniques are similar to computer inkjet printers, however, instead of inks with dyes for paper, the inks used in AJP and PJP contain nanoparticles of metals or ceramics. The inks are precisely placed to form narrow (anywhere from tens to hundreds of micrometers wide) and thin (typically hundreds of nanometers thick) lines and then sintered to create wires and films. These wires and films, when properly arranged, can act as active or passive sensors. Active sensors, such as strain gauges, send a feedback signal that reports the state of the sensor during the test. Passive sensors, such as dosimeters, send no signal, but are examined after the test to determine characteristics of the prior experiment conditions. While in many cases active sensors are considered superior to passive sensors in terms of quantity of data, passive sensors may be easily added to any experiment. They do not require feedthroughs and are often relatively inexpensive.

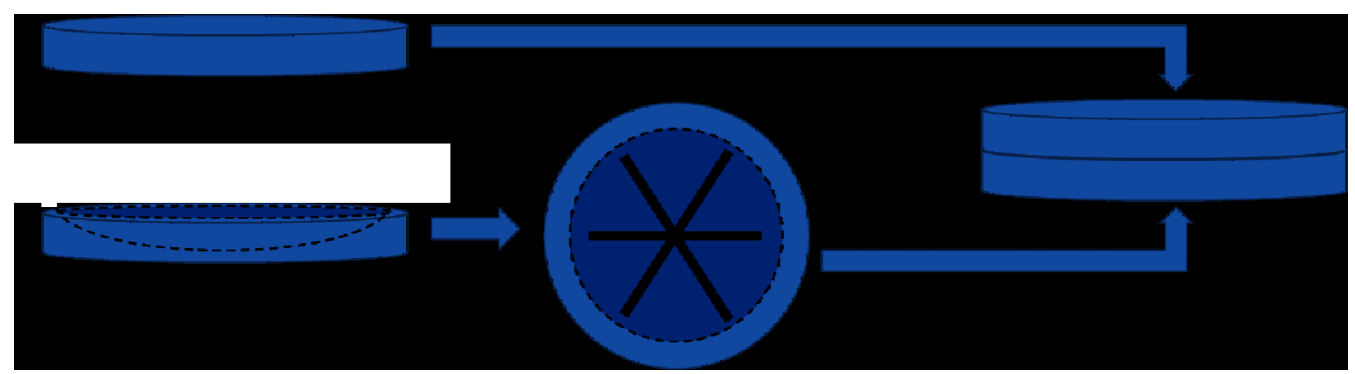

Figure 1. Schematic illustrating the melt wire printing and encapsulation.

Melt wires are a type of passive sensor used to determine peak temperatures during a test. Metal wires of a known material compositions and melting temperatures are positioned in a test. Following the completion of the test, the wires are inspected to determine if the melting temperature was reached, thus informing the researcher that the maximum peak temperature was greater than the melting temperature of the melted wires and lower than the wires that did not melt. Traditional melt wires are commonly used in test reactor experiments such as those at the Advanced Test Reactor (ATR) [1-5]. However, some test designs do not have the space for traditional melt wires. Test capsules that are only a couple millimeters in diameter and are nearly packed full of specimens do not have the space for the wire filaments, which 
are traditionally encapsulated in quartz.

\section{Encapsulation of printed melt wires}

To address this challenge of small spaces, novel melt wire designs are required. A melt wire "chip" was designed, as seen in Fig. 1. A 2-millimeter diameter, 0.5-millimeter thick stainless steel (SS) disk was milled to create a dimple or pocket.

Briefly, a blank SS wafer was placed on the printed side of previously printed melt wire chip, and a laser micro-welding process with a Nd:YAG $(150 \mathrm{~W})$ fiber laser welding system (Fiberstar Workstation 7600, LaserStar Technologies Co., RI, USA; wavelength: $1070 \mathrm{~nm}$, beam diameter: $\geq 25 \mathrm{~mm}$, shield gas: argon) was used, in pulse mode, to form the bond. The optimized parameters included a pulse energy of $0.450 \mathrm{~J}$, pulse length of $3.0 \mathrm{~ms}$, and a pulse frequency of $3.0 \mathrm{~Hz}$. Additionally, by utilizing an overlap method, where each weld spot was aligned to cover $3 / 4$ of the previous weld spot, a continuous seam weld was achieved.

Melt wires were then printed within the pocket using one of the direct ink writing techniques. A second steel disk was then welded overtop using a laser welder. This created a melt wire chip, shown in Fig. 2, that can be easily placed within an experiment, regardless of the environment, as the wires are sealed within the steel.

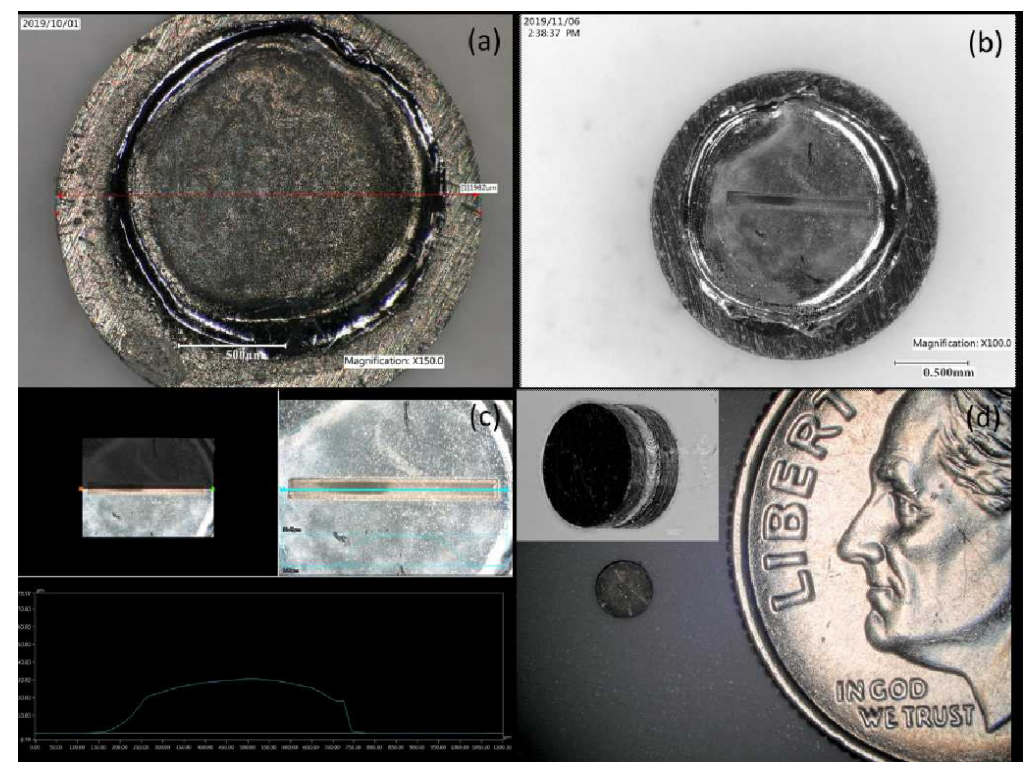

Figure 2. Printing of melt wires: (a) blank steel chip, (b) printed silver melt wires. (c) profilometric analysis of melt wires before sandwiching with a plane steel lid to estimate width and height of the wire, (d) actual $2 \mathrm{~mm}$-diameter melt wire sensor chip. The inset shows clear view of laser welded chip.

While the sealed disks protect the metal wires from corrosive test environments, they make optical observation impossible. X-ray computed tomography (CT) was used to observe the sealed melt.

Validation of the behavior of the printed melt wires was performed in a furnace using a silver wire printed within the chip. The chip was heated to $1,000{ }^{\circ} \mathrm{C}$ to melt the wire and was imaged using X-ray CT before and after melting, as seen in Fig. 3. The wire deformed and coalesced after melting, which is visible in the $\mathrm{X}$-ray CT image.

Once the printing is fully optimized on a test substrate, a 2-mm stainless steel chip is micromachined to ensure the space for printing inside the etched region, which can be seen in the Fig. 3a. The melt ware was then printed inside the chip and characterized to analyze the wire in terms of length, height, weight, 
and morphology (Fig. 3b-c). Later, laser welding was used to sandwich the printed chip with a plain lid for the encapsulation of the melt wire. The actual 2-mm melt wire chip is shown in Fig. 3d. The inset shows the side view of the chip to reveal the welding marks.

\section{Verification of melting behavior using X-ray computed tomography}

Verification was conducted using a "visual" method to identify melting in the printed melt-wires to demonstrate post-irradiation characteristics. X-ray CT is among the most popular visual imaging modalities due to its high-resolution images, fast scan time, low cost, and compatibility with various materials options. It utilizes high-energy electromagnetic radiation to create images of internal structures.

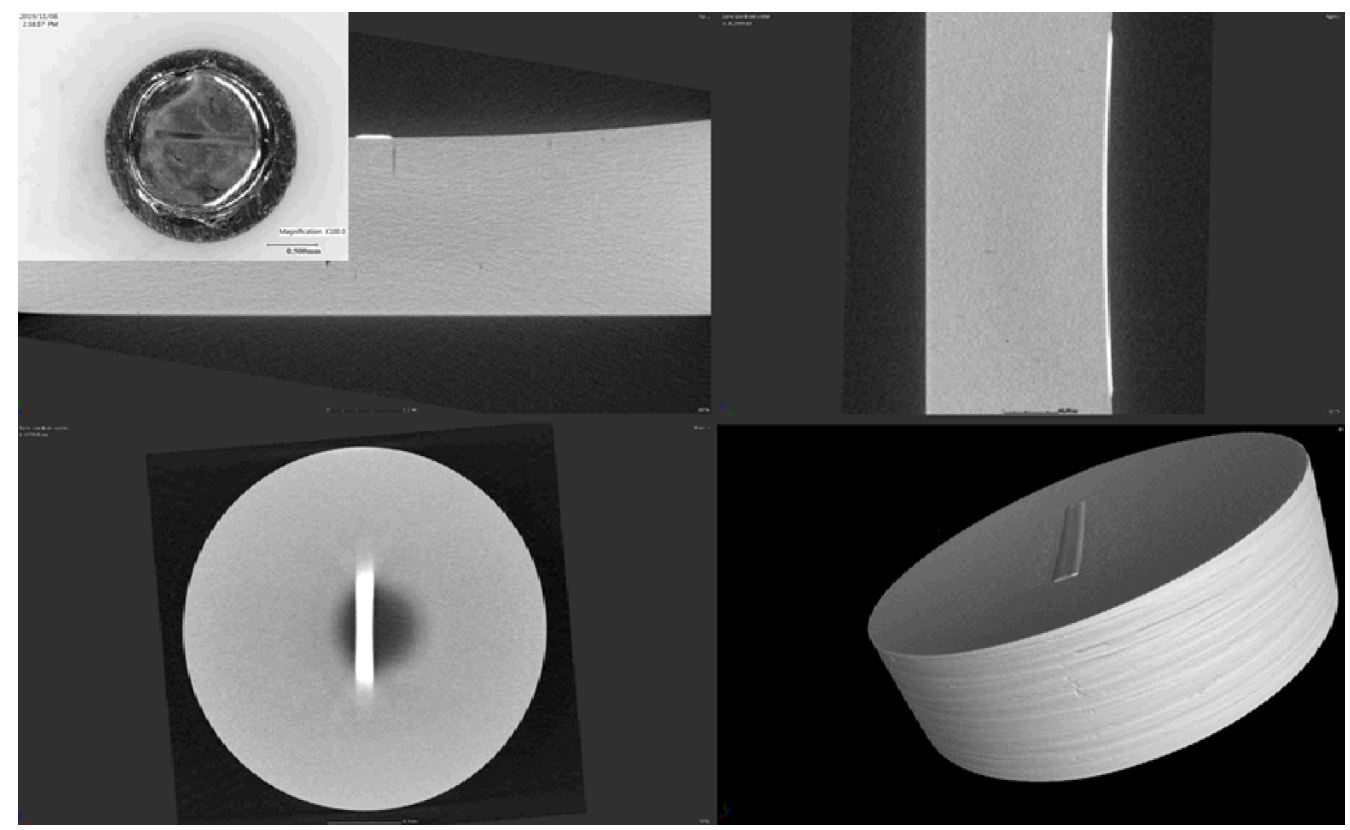

Figure 3. Characterization of printed melt wires. X-ray computer tomography (CT scan) of the melt wires sensor confirms the morphology of the printed line. Inset shows the actual printed chip.

CT scans of nanomaterials require the localization of imaging contrast agents to create contrast, revealing anatomic information. Here, the printed melt wire chips were scanned with nXCT (Nano-focus X-Ray Computed Tomography) to study the morphology before any temperature and welding tests.

A General Electric (GE) Phoenix vitomelx nXCT system was used to collect X-ray radiographs and perform CT reconstruction. The x-ray generator used was a GE $180 \mathrm{kV}$ nano-focus X-ray tube (Model \# XS180NF) with a 5-micron spot size. A GE Dynamic 41-100, flat-panel X-ray detector was used. The detector has a $100 \mu \mathrm{m}$ pixel pitch and an active area of $410 \mathrm{~mm} \times 410 \mathrm{~mm}$. CT was possible through a precision rotation stage located between the source and detector.

The settings for the X-ray generator were $75 \mathrm{kV}$ and $225 \mu \mathrm{A}$, with an aluminum filter that was $0.5 \mathrm{~mm}$ thick. The magnification was $24.99 x$ which resulted in a $4.000176 \mu \mathrm{m}$ Voxel size. A total of 2200 projections were collected resulting in a $0.18^{\circ}$ rotation per projection. Eight exposures were averaged for each projection with a $500.031 \mathrm{~ms}$ timing. GE's proprietary reconstruction software, Phoenix Datos, was used for tomographic reconstruction. 
Using a high temperature furnace and specialized fixtures associated with INL's high temperature test laboratory (HTTL), encapsulated melt wire chips were heated at specified temperatures to ensure melting. The instrumentations were performed to establish the methodology for precise estimation of melting of wires. The melt-wire chips were scanned to compare their morphologies before and after melting to examine melting behavior. This scanning technique involves X-ray scanning of printed and heat-treated samples, performing CT on the X-ray images to build 3D volumetric data, and adaptive segmentation analysis of the 3D data to quantify distinct regions within the sample matrix based on material density and morphology.

The images in Fig. 3 show the morphology of printed silver melt wires in the chip under X-ray scan. Xray computer tomography $(\mathrm{CT})$ was performed on the printed melt wires (Fig. a-c) to ensure the sensors were leak proof and exact melt can be detected. Fig. 4a depicts the fully visible printed melt wire inside the chip in a full X-ray scan.

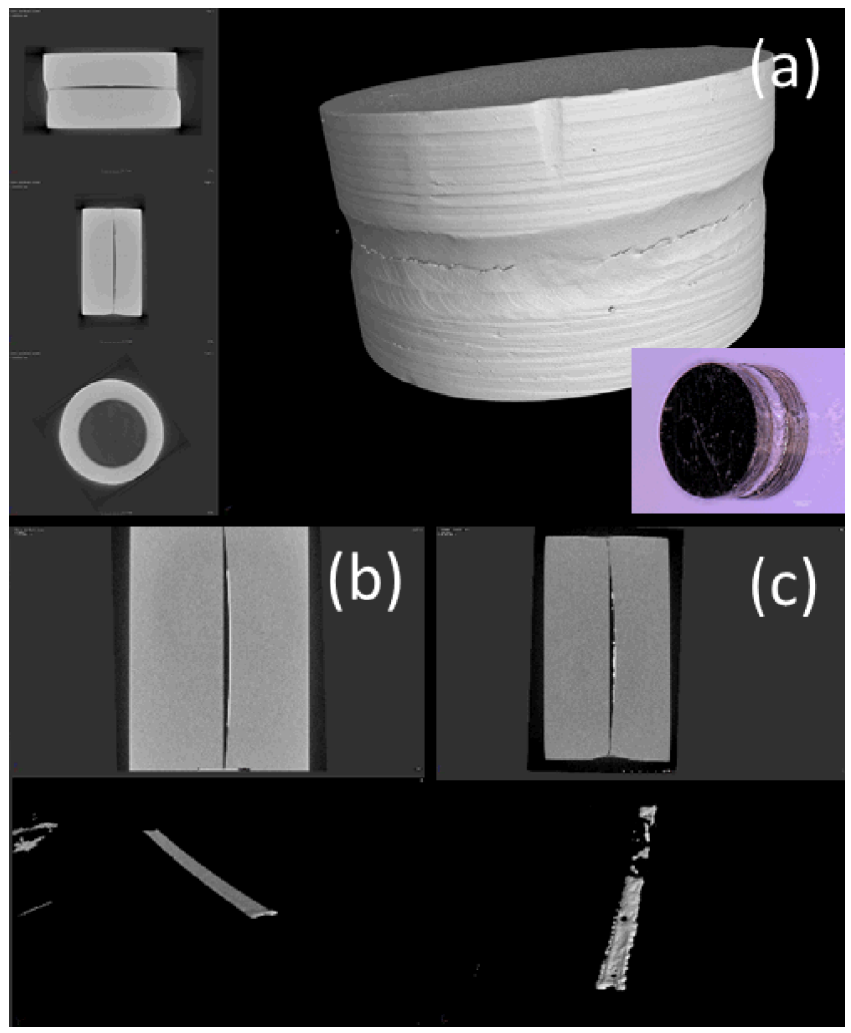

Figure 4. Characterization of printed melt wires (a) X-ray computer tomography (CT scan) of the melt wires sensor to confirm that the line is unharmed due to laser welding. Furnace test of the melt wire: (b) before heating, and (c) after heating at $1000{ }^{\circ} \mathrm{C}$ confirms melting of the printed wire.

The next goal was to check the wires with $\mathrm{CT}$ after furnace heating at $1000{ }^{\circ} \mathrm{C}$ inside an inert atmosphere. Since oxide substrates are not acceptable for radiation tests inside nuclear reactor (because they may oxidize the reactor materials) the chip was prepared with a steel substrate. The X-ray CT scan was performed on the chip after the furnace test and Fig. $\mathbf{4 b - c}$ confirms the melting after comparing with initial morphology. Moreover, data obtained from X-ray CT scans deliver a full $360^{\circ}$ quantitative determination of the fully melted ink, partially melted, and unmelted regions, which is clearly seen in Fig. 4c. The melting of the printed wire was also investigated under an optical microscope. The comparison can be seen in Fig. 5, which confirms the results obtained from CT analysis. Fig. 5a (i-iii) shows the 
printed wire on the 2-mm chip and its area, height and length using optical profilometer, whereas Fig. 5b (i-iii) reveals the same after melting. This report thus describes the methodologies used to apply X-ray CT to interpret experimental results from melting studies of melt wires conducted at INL and highlights some of the key results to confirm melting in the printed wires.

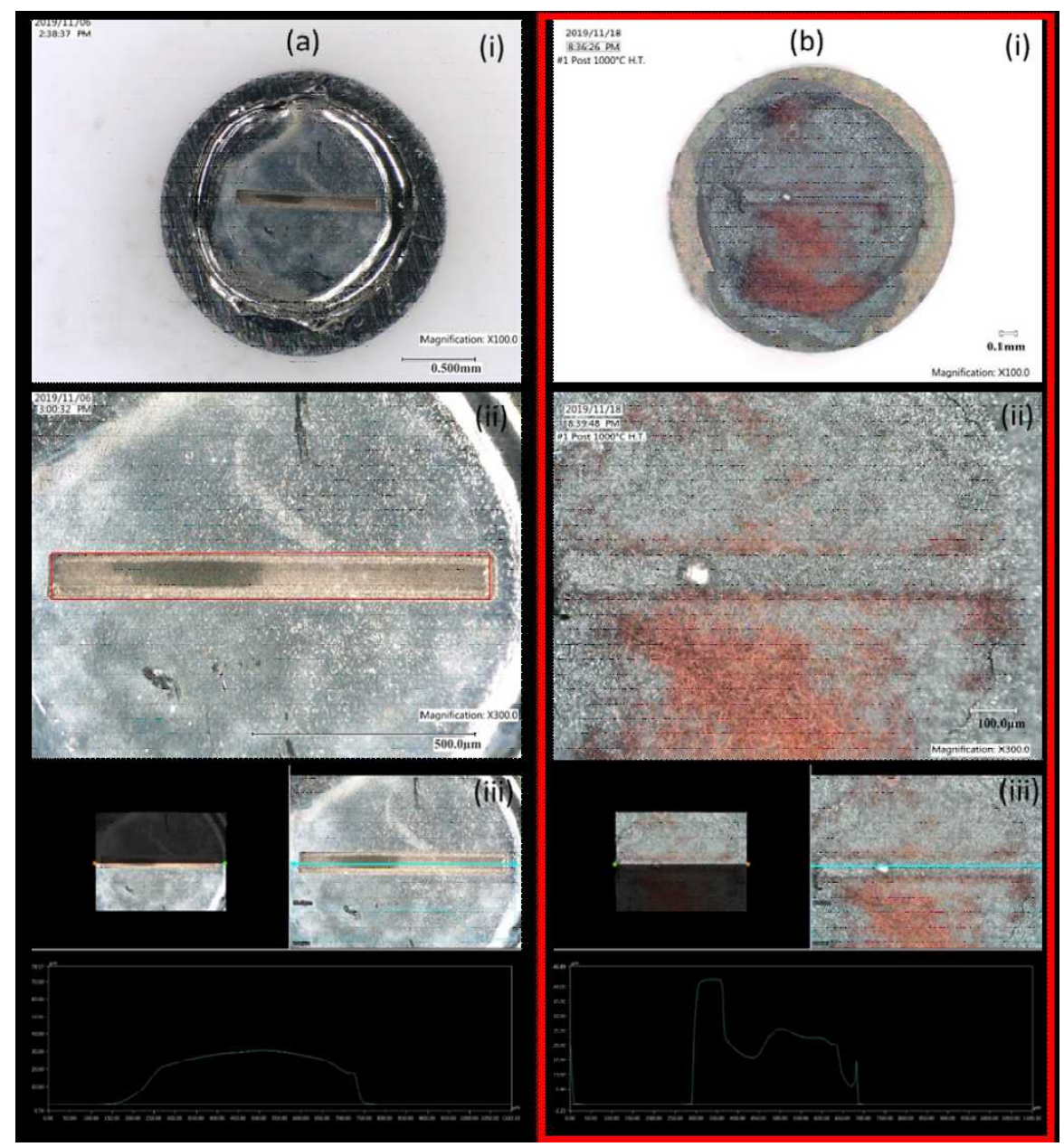

Figure 5. Optical characterization of printed melt wires: (a) before melting. (i) the printed chip, (ii) the clear view and (iii) dimensions of the printed wire using profilometry. (b) after melting due to heating at $1000{ }^{\circ} \mathrm{C}$ confirms the melting. (i) printed chip after melting, (ii) the clear view and (iii) dimensions of the melted portion of the wire using profilometry.

\section{Summary and conclusions}

INL has enhanced its melt wire material capability to support irradiation testing with limited space in the ATR and other test reactors. The miniaturized sensors are two millimeters in diameter, one millimeter thick and can contain multiple melt wires of different compositions. The melt wires are encapsulated to provide protection from potentially corrosive environments. X-ray CT is used to characterize the melt wires and determine the experiment's peak temperature. Testing has been performed outside of test reactors to validate the manufacturing procedures and ability to visualize the melted wires ranging from $85-1455{ }^{\circ} \mathrm{C}$. The first insertion of a melt wire chip into a test reactor is expected to occur in the Fall of 2020. 


\section{Acknowledgement}

This work was supported through the Department of Energy In-Pile Instrumentation program, under DOE Idaho Operations Office Contract DE-AC07-05ID14517.

\section{References}

1. K. L. Davis, D.L. Knudson, J.E. Daw, J.L.Rempe and A.J. Palmer, "Melt Wire Sensors Available to Determine Peak Temperatures in ATR Irradiation Testing," 8th International Topical Meeting on Nuclear Plant Instrumentation, Control, and Human Machine Interface Technologies (NPIC\&HMIT2012), San Diego, CA, July 22-26, 2012.

2. K. L. Davis and D. L. Knudson, "Specimen Melt Temperatures for Use in the Advanced Test Reactor," TEV-1175, March 2011.

3. K.L. Davis, "Melt Wire Testing," INL Interoffice Memorandum, October 14, 2011.

4. L. Rempe, D. L. Knudson, J. E. Daw, T. C. Unruh, B. M. Chase, K. L. Davis, A. J. Palmer, and R. S. Schley, "Advanced in-pile instrumentation for material and test reactors," in Advancements in Nuclear Instrumentation Measurement Methods and their Applications (ANIMMA), 2013 3rd International Conference on, 2013, pp. 1-11, DOI: 10.1109/ANIMMA.2013.6728013.

5. Joy L. Rempe, Darrell L. Knudson, Joshua E. Daw, Troy Unruh, Benjamin M. Chase, Joe Palmer, Keith G. Condie, and Kurt L. Davis, "Enhanced In-pile Instrumentation at the Advanced Test Reactor," IEEE, 2011. 